\title{
Heat transfer in rough-wall turbulent thermal convection in the ultimate regime
}

\author{
Michael MacDonald, ${ }^{1, *}$ Nicholas Hutchins, ${ }^{1}$ Detlef Lohse, ${ }^{2,3}$ and Daniel Chung ${ }^{1, \dagger}$ \\ ${ }^{1}$ Department of Mechanical Engineering, University of Melbourne, Victoria 3010, Australia \\ ${ }^{2}$ Physics of Fluids Group, MESA+ Institute, J. M. Burgers Center for Fluid Dynamics and Max Planck Center \\ Twente, University of Twente, P.O. Box 217, 7500AE Enschede, The Netherlands \\ ${ }^{3}$ Max Planck Institute for Dynamics and Self-Organization, 37077 Göttingen, Germany
}

(Received 12 December 2018; published 22 July 2019)

\begin{abstract}
Heat and momentum transfer in wall-bounded turbulent flow, coupled with the effects of wall roughness, is one of the outstanding questions in turbulence research. In the standard Rayleigh-Bénard problem for natural thermal convection, it is notoriously difficult to reach the so-called ultimate regime in which the near-wall boundary layers are turbulent. Following the analyses proposed by Kraichnan [Phys. Fluids 5, 1374 (1962)] and Grossmann and Lohse [Phys. Fluids 23, 045108 (2011)], we instead utilize recent direct numerical simulations of forced convection over a rough wall in a minimal channel [MacDonald et al., J. Fluid Mech. 861, 138 (2019)] to directly study these turbulent boundary layers. We focus on the heat transport (in dimensionless form, the Nusselt number $\mathrm{Nu}$ ) or equivalently the heat transfer coefficient (the Stanton number $C_{h}$ ). Extending the analyses of Kraichnan and Grossmann and Lohse, we assume logarithmic temperature profiles with a roughness-induced shift to predict an effective scaling of $\mathrm{Nu} \sim \mathrm{Ra}^{0.42}$, where $\mathrm{Ra}$ is the dimensionless temperature difference, corresponding to $C_{h} \sim \mathrm{Re}^{-0.16}$, where $\mathrm{Re}$ is the centerline Reynolds number. This is pronouncedly different from the skin-friction coefficient $C_{f}$, which in the fully rough turbulent regime is independent of Re, due to the dominant pressure drag. In rough-wall turbulence, the absence of the analog to pressure drag in the temperature advection equation is the origin for the very different scaling properties of the heat transfer as compared to the momentum transfer. This analysis suggests that, unlike momentum transfer, the asymptotic ultimate regime, where $\mathrm{Nu} \sim$ $\mathrm{Ra}^{1 / 2}$, will never be reached for heat transfer at finite Rayleigh number.
\end{abstract}

DOI: 10.1103/PhysRevFluids.4.071501

Introduction. Heat transfer in wall-bounded turbulent flow is one of the outstanding problems in turbulence, from both fundamental and applied points of view. The canonical system to study it is Rayleigh-Bénard (RB) convection [1-3], i.e., the flow in a container heated from below and cooled from above. Here the key questions are as follows: How does the heat transfer (in dimensionless form, the Nusselt number $\mathrm{Nu}$ ) scale with the temperature difference between the top and bottom walls (in dimensionless form, the Rayleigh number Ra)? And how does this scaling change with wall roughness? For smooth walls in the so-called classical regime, in which the boundary layers (BLs) are of Prandtl-Blasius (i.e., basically laminar) type, the dependencies are reasonably understood along the unifying theory of thermal convection [1,4-6]. However, the situation is much less clear in the so-called ultimate regime, in which the BLs become turbulent [7-10] and the heat transfer is thus

\footnotetext{
*Present address: Jet Propulsion Laboratory, California Institute of Technology, Pasadena, CA 91109, USA.

†daniel.chung@unimelb.edu.au
} 
enhanced. In this regime, Kraichnan [7] predicted that $\mathrm{Nu} \sim \mathrm{Ra}^{1 / 2}[\log (\mathrm{Ra})]^{-3 / 2}$. Later, Grossmann and Lohse $[9,10]$ used logarithmic velocity and temperature profiles to quantify the logarithmic correction term. Beyond the transition, which for gases was predicted [5] to occur around $\mathrm{Ra} \sim$ $10^{14}$, both predictions imply an effective scaling $\mathrm{Nu} \sim \mathrm{Ra}^{\gamma_{\text {eff }}}$ with $\gamma_{\text {eff }} \approx 0.38$. As $\mathrm{Ra} \rightarrow \infty$, the logarithmic correction terms become negligible and the flow approaches the so-called asymptotic ultimate regime where $\mathrm{Nu} \sim \mathrm{Ra}^{1 / 2}$. This asymptotic ultimate regime implies that viscosity and thermal diffusivity effects have a negligible impact on the flow. In contrast to [7-9], Owen and Thompson [11] proposed that the asymptotic ultimate (and upper bound [12-14]) scaling exponent $1 / 2$ is never achieved.

Whether and when the transition to the ultimate regime indeed occurs, and to what turbulent state, has been hotly debated in the community. While Ahlers et al. experimentally found such a transition around $\mathrm{Ra} \sim 10^{14}$ [15-17], Chavanne, Roche, et al. [18-20] observed it at lower $\mathrm{Ra} \sim 10^{11}-10^{12}$, and others still do not find such a transition at all [21-23]. To clarify this question, major numerical efforts are undertaken to solve the underlying Boussinesq equations in this large-Ra regime. While in three dimensions (3D) the required computational power is presently prohibitive, in two dimensions (2D) the onset of such a transition has recently been observed around $\mathrm{Ra} \sim 10^{13}$ [24,25], namely in the effective scaling of $\mathrm{Nu}(\mathrm{Ra})$ and in the structure of the BLs, changing toward a logarithmic profile in the ultimate regime.

To trigger the onset of the ultimate regime, i.e., the transition from a laminar-type BL to a turbulent one, various wall-roughness elements have been employed, both in experiments [26-35] and in numerical simulations [36-39]. In general, these efforts have led to an enhanced Nu versus $\mathrm{Ra}$ scaling in some intermediate-Ra regime, and in limited-Ra regimes even an effective Nu versus $\mathrm{Ra}$ scaling exponent of $1 / 2$ can be achieved. For large Ra (but still below the onset of the ultimate regime), the effective scaling exponent settles back to a value close to the one in the classical regime [39], as then the thermal sublayer is thinner than the roughness elements and starts to conform to the roughness topography. Only for even larger Ra-hitherto not yet achieved in rough-wall RB flow-is the transition toward a turbulent BL throughout, and enhanced $\mathrm{Nu}$ versus $\mathrm{Ra}$, expected.

To address the question of heat transport in smooth and rough-wall RB convection in the ultimate regime, in this paper we will assume the hypothesis proposed by Kraichnan [7] and Grossmann and Lohse $[9,10]$ that the boundary layers are turbulent with logarithmic profiles. This allows us to employ our understanding of heat transfer in smooth and in particular rough-wall fully turbulent forced-convection channel flow. The advantage of such flow is that the driving is mechanically supplied (namely by shear), which is much more efficient than the thermal driving in RB flow. Therefore, in numerical simulations the transition to turbulence in the boundary layers - manifesting itself in a logarithmic velocity profile — can easily be achieved [40,41]. Such turbulent boundary layers with a logarithmic velocity profile also exist in the shear-driven Taylor-Couette (TC) flow [42], which is viewed as the "twin" of RB flow [43]. For that flow, indeed the ultimate regime with the corresponding Nusselt number $\mathrm{Nu}_{\omega}$ (the dimensionless angular velocity transport [44]) scaling $\mathrm{Nu}_{\omega} \propto \mathrm{Ta}^{0.38}$ (where the Taylor number Ta is the dimensionless mechanical driving strength) can be achieved both in experiments and in numerical simulations; see the review article [42]. In TC flow with a rough wall, even the asymptotic ultimate regime $\mathrm{Nu}_{\omega} \propto \mathrm{Ta}^{1 / 2}$ can be achieved, both experimentally [45-47] and numerically [47]. This regime corresponds to fully rough pipe or channel flow in which the friction factor becomes Reynolds number independent [47-50]. The reason is that in this regime the drag is determined by the pressure drag, and shear (viscous) drag hardly plays a role. However, this dominant pressure drag also implies that the analogy between heat transfer in RB and angular velocity transport in TC breaks down for roughness, due to the lack of a pressure-like component in the temperature advection equations [39]. The quantitative effect of roughness on the heat transfer in RB flows, despite this qualitative understanding, is therefore not well understood, and we will address it here using forced convection channel flow. Note that, like $\mathrm{RB}$ and TC flows, numerical simulations of closed-channel (and pipe) flows employing periodic boundary conditions in the flow direction also enjoy exact energy balances [51]. 

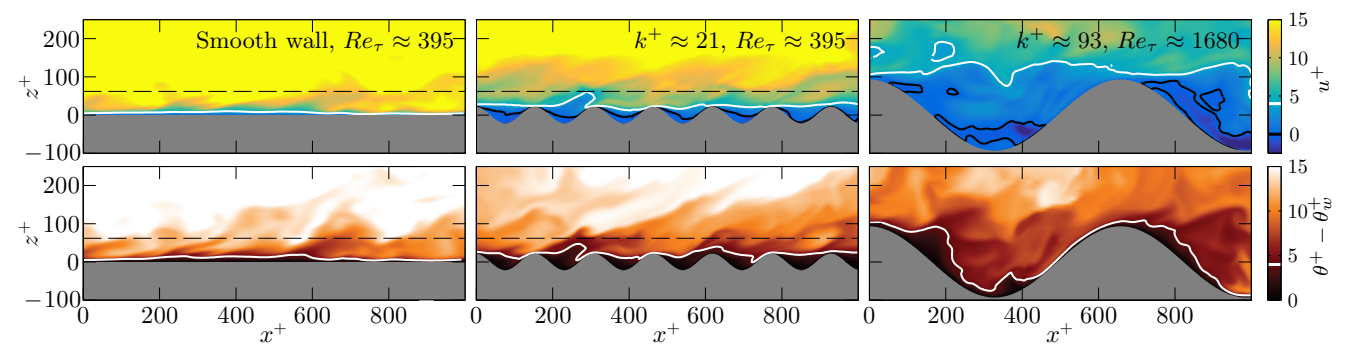

FIG. 1. Instantaneous steamwise velocity (top) and temperature (bottom) for smooth-wall (left), transitionally rough (center), and fully rough (right) flows, from forced convection DNS [52]. The horizontal dashed line shows the minimal channel critical height, $z_{c}=0.4 L_{y}$. The black contour line shows zero velocity (recirculation), while the white contour line shows the value $u^{+}=4$ and $\theta^{+}-\theta_{w}^{+}=4$, to highlight the viscous and thermal diffusive sublayers. Flow is from left to right.

In the present work, we will use the recent rough-wall turbulent forced convection results from Ref. [52] as a model for the near-wall shear-dominated turbulent boundary layers observed in highRa natural convection flows, as envisioned by Kraichnan [7]. We will therefore seek to quantify and explain the effect of roughness on the scaling exponent of the Nusselt number in the ultimate regime. This involves extending the analysis of Ref. [9] for smooth-wall ultimate RB flow with logarithmic velocity and temperature profiles, by quantifying the shift in the profiles induced by the roughness.

Briefly, we summarize the forced convection direct numerical simulations (DNSs) of Ref. [52], in which buoyancy forces were neglected so that temperature was a passive scalar. Periodic boundary conditions were employed in the streamwise $(x)$ and spanwise $(y)$ directions and no-slip, impermeability, and isothermal $\left(\theta_{w}=0\right)$ conditions were applied to the top and bottom walls, with $z$ denoting the wall-normal (vertical) direction. A body forcing to the momentum equation was used to drive the flow at constant bulk velocity through the channel. An internal heating body force to the energy equation was used for temperature, representing a hot fluid being cooled by the walls. The Prandtl number was set to that of air at room temperature, $\operatorname{Pr} \equiv v / \kappa=0.7$, where $v$ is the kinematic viscosity and $\kappa$ is the thermal diffusivity. Different friction Reynolds numbers, $\operatorname{Re}_{\tau}=U_{\tau} h / v$, were simulated with $395 \lesssim \operatorname{Re}_{\tau} \lesssim 1680$, where $U_{\tau}$ is the friction velocity and $h$ is the channel half height, defined for the rough-wall flow to be distance between the channel center and the roughness mean height, corresponding to the hydraulic half-height [53]. Three-dimensional sinusoidal roughness with semiamplitude of either $k=h / 18$ or $k=h / 36$ and wavelength $\lambda_{x}=\lambda_{y}=\lambda \approx 7.07 k$ was applied to both the bottom and top walls. As the flow speed and friction Reynolds number increase, the roughness Reynolds number $k^{+}=k U_{\tau} / v$ increases toward the fully rough regime. Superscript + indicates nondimensionalization on $v, U_{\tau} \equiv \sqrt{\tau_{w} / \rho}$, and the friction temperature $\Theta_{\tau} \equiv\left[q_{w} /\left(\rho c_{p}\right)\right] / U_{\tau}$, with $\tau_{w}$ and $q_{w}$ being the temporally and spatially averaged momentum and heat fluxes at the wall, $\rho$ being the fluid density, and $c_{p}$ being the specific heat at constant pressure. The minimal-span channel for rough wall flows was used [54,55], in which the spanwise domain width is purposely very narrow and only the near-wall turbulent flow is captured up to a critical height $z_{c} \approx 0.4 L_{y}$, where $L_{y}$ is the channel span. Smooth-wall channel simulations with matched channel domain sizes were also conducted to ensure that the differences between the smooth- and rough-wall flows were due to the roughness alone and not the channel span.

Figure 1 shows instantaneous snapshots of the streamwise velocity and fluid temperature from the simulations of Ref. [52]. A white contour line for $u^{+}=4$ and $\theta^{+}-\theta_{w}^{+}=4$ has been selected to provide an indication of the viscous and thermal diffusive sublayers. The smooth-wall flow produces thin viscous and thermal sublayers close to the wall that appear similar, although the thermal sublayer is slightly larger due to the Prandtl number being less than unity. The roughness in the transitionally rough regime $\left(k^{+} \approx 21\right)$ produces much thicker sublayers and, like the smooth wall, appear similar between the velocity and temperature fields. The final (rightmost) panel shows 
much larger roughness that is nominally fully rough $\left(k^{+} \approx 93\right)$. The selected contour line of $u^{+}=4$ resides mostly above the roughness crests, indicating the fluid below the level of the roughness crests is nearly stationary due to the increased dominance of pressure drag. Conversely, the thermal diffusive sublayer is thin and closely follows the roughness geometry; it appears more like that of the smooth wall if the wall were contorted to match the roughness geometry.

Turbulent boundary layers in the rough-wall ultimate regime. The turbulent boundary layers observed in high-Ra (ultimate regime) natural convection flows are characterized by local buoyancy forces that are much smaller than the shear forces. This leads to mean velocity and temperature profiles that are logarithmic functions of distance from the wall [9], the same as in forced convection flows [56-58], given as

$$
\begin{gathered}
\bar{u}^{+} \equiv \frac{\bar{u}}{U_{\tau}}=\frac{1}{\kappa_{m}} \log \left(z^{+}\right)+A_{m}-\Delta U^{+}\left(k^{+}\right), \\
\bar{\theta}^{+}-\bar{\theta}_{w}^{+} \equiv \frac{\bar{\theta}-\bar{\theta}_{w}}{\Theta_{\tau}}=\frac{1}{\kappa_{h}} \log \left(z^{+}\right)+A_{h}(\operatorname{Pr})-\Delta \Theta^{+}\left(k^{+}\right),
\end{gathered}
$$

where $\kappa_{m} \approx 0.4$ is the von Kármán constant, which is slightly larger for heat transfer with $\kappa_{h} \approx 0.46$ due to the turbulent Prandtl number (the ratio of momentum and heat transfer eddy diffusivities) being below unity [58,59]. As in Ref. [52], we take the smooth-wall offsets to be $A_{m} \approx 5.0$ and $A_{h}(\operatorname{Pr}=0.7) \approx 3.2$. The enhanced skin friction and heat transfer of roughness lead to a downward shift of these logarithmic profiles, represented by the roughness function, $\Delta U^{+}[49]$, and temperature difference, $\Delta \Theta^{+}$[52,59-61]. These two quantities are a flow property of a given rough surface and vary with the roughness Reynolds number. Evaluating Eqs. (1) and (2) at the middle of an RB cell, $z=L / 2$, yields

$$
\begin{gathered}
U^{+} \equiv \bar{u}^{+}\left(z=\frac{L}{2}\right)=\frac{1}{\kappa_{m}} \log \left(\frac{\frac{1}{2} \operatorname{Re}}{U^{+}}\right)+A_{m}-\Delta U^{+}\left(\frac{k}{L} \frac{\operatorname{Re}}{U^{+}}\right), \\
\Theta^{+} \equiv \bar{\theta}^{+}\left(z=\frac{L}{2}\right)-\bar{\theta}_{w}^{+}=\frac{1}{\kappa_{h}} \log \left(\frac{\frac{1}{2} \operatorname{Re}}{U^{+}}\right)+A_{h}(\operatorname{Pr})-\Delta \Theta^{+}\left(\frac{k}{L} \frac{\operatorname{Re}}{U^{+}}\right),
\end{gathered}
$$

where the Reynolds number $\operatorname{Re}=U L / \nu$. Equations (3) and (4) thus describe $U^{+}$and $\Theta^{+}$for a given flow state governed by $\operatorname{Re}$ and $\operatorname{Pr}$ and by the relative roughness $k / L$, provided $\Delta U^{+}$and $\Delta \Theta^{+}$ are known.

We define the skin-friction coefficient as $C_{f} \equiv \tau_{w} /\left(\frac{1}{2} \rho U^{2}\right)=2 / U^{+2}$ and heat-transfer coefficient (Stanton number) as $C_{h} \equiv q_{w} /\left(\rho c_{p} U\right)=1 /\left(U^{+} \Theta^{+}\right)$. The temperature profiles from the top and bottom walls must match at the centerline so that $2 \Theta=\Delta$, where $\Delta$ is the driving temperature difference in RB domains, and thus we define the Nusselt number as $\mathrm{Nu}=\operatorname{Pr} \operatorname{Re} /\left(U^{+} \Delta^{+}\right)=$ $\operatorname{Pr} \operatorname{Re} C_{h} / 2$. In order to describe the rough-wall $C_{f}, C_{h}$, and $\mathrm{Nu}$ as a function of Reynolds number, we therefore require knowledge of $\Delta U^{+}$and $\Delta \Theta^{+}$. Note that for the smooth-wall case $\left(\Delta U^{+}=\right.$ $\Delta \Theta^{+}=k / L=0$ ), Eq. (3) yields the implicit Prandtl-von Kármán logarithmic skin-friction law, which can be solved using Lambert's $\mathcal{W}$ function with $U^{+}=\left(1 / \kappa_{m}\right) \mathcal{W}\left(\operatorname{Re} \kappa_{m} \exp \left(A_{m} \kappa_{m}\right) / 2\right)$. Grossmann and Lohse [9] obtained the smooth-wall ultimate-regime Nusselt number scaling exponent of $\gamma_{\text {eff }} \approx 0.38$ using this result.

Figure 2(a) shows a sketch of a sinusoidal rough-wall RB domain. We can obtain the roughness function $\Delta U^{+}$and temperature difference $\Delta \Theta^{+}$from the turbulent forced convection system of Ref. [52]. These two quantities are shown in Fig. 2(b), as a function of the equivalent sand-grain roughness Reynolds number, $k_{s}^{+} \approx 4.1 k^{+}$. This scaling guarantees a collapse of the roughness function with that of Nikuradse's sand-grain data in the fully rough regime (here $k_{s}^{+} \gtrsim 150$ ), where $\Delta U_{F R}^{+}=\left(1 / \kappa_{m}\right) \log \left(k_{s}^{+}\right)+A_{m}-C_{n}$ (blue dashed line), with $C_{n} \approx 8.5$ being Nikuradse's constant $[48,62]$. Within the asymptotic fully rough regime, viscous effects are negligible and the pressure (or form) drag is dominant $[47,63,64]$. Note that $k_{s}$ must be determined dynamically for a given rough 

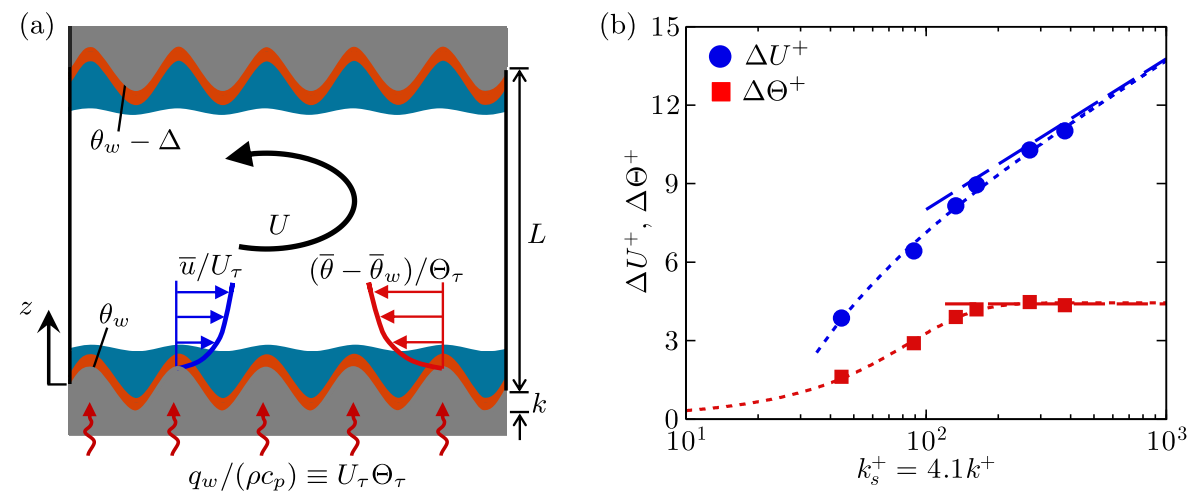

FIG. 2. (a) Sketch of the Rayleigh-Bénard system with roughness. Under fully rough conditions, the viscous sublayer (blue) is larger than the thin, roughness-conforming thermal sublayer (orange) (Fig. 1). The near-wall velocity and temperature profiles are logarithmic in the ultimate regime [7,9] but, relative to a smooth wall, are shifted by $\Delta U^{+}$and $\Delta \Theta^{+}$, respectively, due to the roughness Eqs. (1) and (2). (b) Roughness function, $\Delta U^{+}$(blue), and temperature difference, $\Delta \Theta^{+}$(red), as a function of the equivalent sand-grain roughness Reynolds number, $k_{s}^{+} \approx 4.1 k^{+}$. Symbols are the forced convection DNS data [52]; dotted lines are curve fits to the DNS data (see text); dashed lines are the fully rough asymptotes.

surface and is not a simple geometric length scale of the roughness. The temperature difference, meanwhile, is tending toward a constant value of $\Delta \Theta_{F R}^{+} \approx 4.4$ in the fully rough regime (red dashed line). Like $k_{s}$, the exact value of $\Delta \Theta_{F R}^{+}$is a dynamic parameter that is likely to be roughness dependent. Ultimately however, with this information, in the fully rough regime Eqs. (3) and (4) become

$$
\begin{gathered}
U_{F R}^{+}=C_{n}-\frac{1}{\kappa_{m}} \log \left(\frac{k_{s}}{L / 2}\right), \\
\Theta_{F R}^{+}=\frac{1}{\kappa_{h}} \log \left(\frac{\frac{1}{2} \operatorname{Re}}{U_{F R}^{+}}\right)+A_{h}-\Delta \Theta_{F R}^{+} .
\end{gathered}
$$

That is, the friction-normalized centerline velocity is constant and only depends on the relative roughness $k_{s} / L$, while the centerline friction-normalized temperature remains dependent on the Reynolds number.

The dotted lines in Fig. 2(b) are curve fits to the DNS data. For the roughness function, we use the fit from Ref. [65] with $\Delta U_{\text {fit }}^{+} \approx\left(1 / \kappa_{m}\right) \log \left(k_{s}^{+}\right)+A_{m}-C_{n}-\left(a / k_{s}^{+}\right)^{b}$, where $a \approx 89.3$ and $b \approx$ 1.12. While the fitting constants are different from those of Ref. [65] due to the different roughness geometries, this function correctly tends toward the fully rough asymptote for large $k_{s}^{+}$. However, the function quickly reaches zero at $k_{s}^{+} \approx 25$, much more rapidly than for sinusoidal roughness or sand-grain roughness (see Fig. 5 of [52]). We therefore only use $\Delta U_{f i t}^{+}$for $k_{s}^{+} \gtrsim 35$. For the temperature difference, a sigmoid function across the entire range of $k_{s}^{+}$is used, with $\Delta \Theta_{\text {fit }}^{+} \approx B+$ $K /\left[1+\exp \left(c+d k_{s}^{+}\right)\right]$, where $B \approx-1.66, K \approx 6.11, c \approx 0.97$, and $d \approx-0.0239$. This function correctly goes to zero for small $k_{s}^{+}$(i.e., a smooth wall) and tends toward $\Delta \Theta_{F R}^{+} \approx 4.4$ in the limit of $k_{s}^{+} \rightarrow \infty$ (i.e., the fully rough regime). We can therefore obtain $C_{f}, C_{h}$, and $\mathrm{Nu}$ numerically using $\Delta U_{\text {fit }}^{+}$and $\Delta \Theta_{\text {fit }}^{+}$with Eqs. (3) and (4), although the functional form of $\Delta U_{\text {fit }}^{+}$precludes an analytical solution for $U^{+}$.

Effective heat-transfer scaling in the ultimate regime. Figures 3(a) and 3(b) show the skin-friction and heat-transfer coefficients as a function of Reynolds number. Here, the solid lines show the smooth-wall $C_{f}$ and $C_{h}$ calculated using the logarithmic velocity and temperature profiles [Eqs. (3) and (4) with $\left.\Delta U^{+}=\Delta \Theta^{+}=0\right]$. The dotted lines correspond to the rough-wall $C_{f}$ and $C_{h}$ calculated 

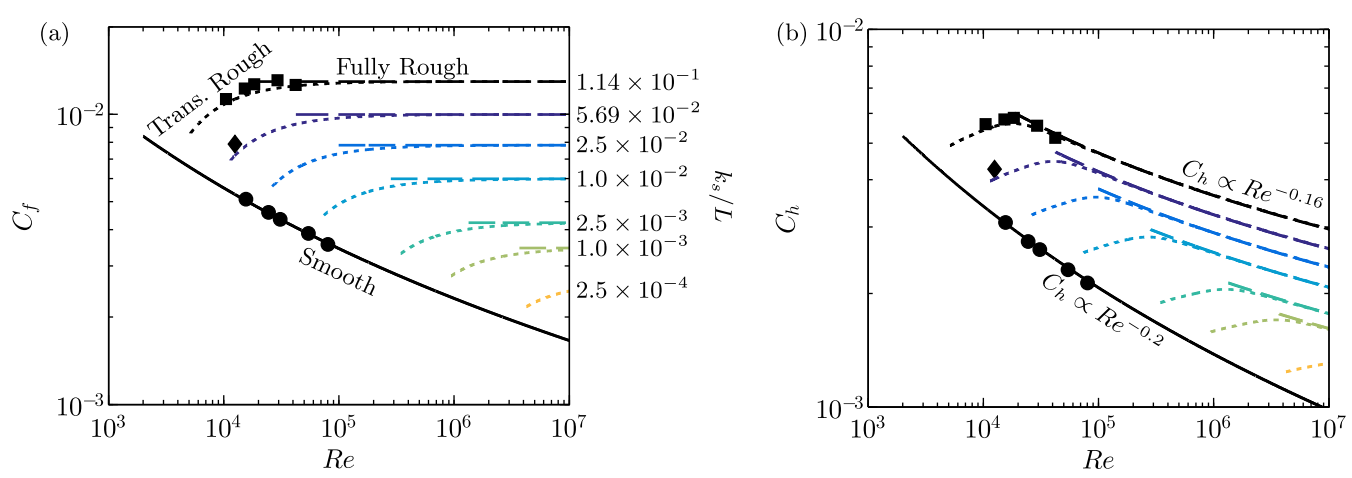

FIG. 3. (a) Skin-friction coefficient and (b) heat-transfer coefficient (Stanton number) against Reynolds number. DNS data [52] shown by symbols: circles, smooth wall; squares, rough wall $k_{s} / L=1.14 \times 10^{-1}$; diamond, $k_{s} / L=5.69 \times 10^{-2}$. Line styles: solid, smooth wall [Eqs. (3) and (4) with $\Delta U^{+}=\Delta \Theta^{+}=0$ ]; dotted, transitionally rough regime [Eqs. (3) and (4) with $\Delta U_{\text {fit }}^{+}$and $\Delta \Theta_{\text {fit }}^{+}$curve fits from Fig. 2(b)]; dashed, fully rough regime [Eqs. (5) and (6)]. Different colors (in both figures) correspond to different relative roughnesses $k_{s} / L$, with legend in panel (a).

using Eqs. (3) and (4) with the curve fits $\Delta U_{\text {fit }}^{+}$and $\Delta \Theta_{\text {fit }}^{+}$from Fig. 2(b), while the dashed lines show the asymptotic fully rough regime [Eqs. (5) and (6)]. The relative roughness ratio $k_{s} / L$ is varied, given by the different colors, where it is assumed $\Delta U^{+}$and $\Delta \Theta^{+}$are independent of $k_{s} / L$. The symbols are the DNS data [52], assuming that the channel centerline $h$ is equal to the middle of the RB cell, $L / 2$. For the smooth-wall flow, both of these coefficients monotonically reduce with Reynolds number at the same rate. Roughness enhances momentum and thermal transfer, leading to an increase in these coefficients relative to the smooth wall. In the fully rough regime $(\operatorname{Re} \gtrsim 2 \times$ $10^{4}$ for $k_{s} / L=1.14 \times 10^{-1}$, black dashed line), the skin-friction coefficient becomes constant with Reynolds number, with $C_{f} \approx 0.013$. This indicates that the viscous effects are negligible and the momentum transfer has attained an asymptotic state [39]. Conversely, the heat-transfer coefficient reduces with Reynolds number in the fully rough regime, similar to the smooth wall, and does not appear to reach any asymptotic state. At the present Reynolds numbers, Eqs. (3)-(6) predict $C_{h} \propto \mathrm{Re}^{-0.2}$ for the smooth wall (e.g., Ref. [66]), while for the rough wall with $k_{s} / L=1.14 \times 10^{-1}$ it scales as $C_{h} \propto \mathrm{Re}^{-0.16}$.

Ultimately, we would like to know the dependency of the Nusselt number on the Rayleigh number, $\mathrm{Nu} \sim \mathrm{Ra}^{\gamma_{\text {eff }}}$. However, the log equations [Eqs. (3)-(6)] as well as the forced convection DNS data are functions of the Reynolds number, requiring an assumption of the form $\operatorname{Re}=A \mathrm{Ra}^{\beta}$ in order to determine the Rayleigh number dependency. Grossmann and Lohse [9] made the same assumption in their work, where they used $\mathrm{Re}=0.346 \mathrm{Ra}^{0.443}$, corresponding to boundary layers that were not yet turbulent. As we are explicitly dealing with turbulent (logarithmic) boundary layers, we use the ultimate-regime scaling of $\beta=1 / 2$ [9]. More recently, Shishkina et al. [67] derived this relation with $\beta=1 / 2$ from the Prandtl BL equations with very weak assumptions. We take the coefficient $A=0.7$, which results in $\mathrm{Nu}\left(\mathrm{Ra}=10^{14}\right) \approx 2500$ for the smooth wall, in agreement the laboratory experiments of Ref. [16]. The exact choice of $A$ and $\beta$ will alter the absolute values of $\mathrm{Nu}$ and $\gamma_{\text {eff }}$ for a given $\mathrm{Ra}$; however, we emphasize that the assumption made here does not alter the main conclusions of this paper.

Figure 4(a) shows the Nusselt number as a function of the Rayleigh number. The inset highlights just the DNS data, where the smooth-wall forced convection data (circles) have an effective scaling exponent of $\gamma_{\text {eff }} \approx 0.38$ (solid black line), matching that observed in ultimate RB convection [9], as expected. Note that the mechanically supplied shear of forced convection corresponds to a very strong wind in RB convection, explaining the relatively low Ra values in the inset of Fig. 4(a). A realistic RB flow in the ultimate regime typically has higher $\mathrm{Ra}$ values due to a weaker wind 

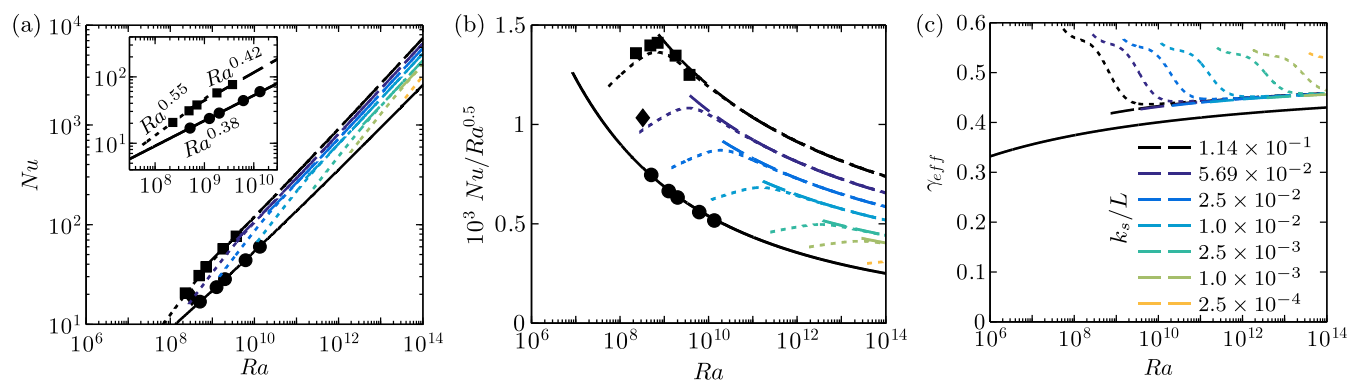

FIG. 4. (a) Nusselt number, $\mathrm{Nu}$, (b) compensated $\mathrm{Nu} / \mathrm{Ra}^{0.5}$, and (c) predicted (from log equations) effective scaling exponent, $\gamma_{\text {eff }}$, in $\mathrm{Nu} \propto \mathrm{Ra}^{\gamma_{\text {eff }}}$, as as function of the Rayleigh number. The inset in panel (a) highlights just the forced convection DNS data [52]. Symbols and line styles are the same as in Fig. 3(a).

(i.e., a lower Reynolds number, or coefficient $A$ in the assumed Re-Ra relationship above). The transitionally rough regime, meanwhile, has an enhanced exponent of $\gamma_{\mathrm{eff}} \approx 0.55$ shown by the dotted lines (similar to the rough-wall RB experiments by Ref. [34]); however, in the asymptotic fully rough regime the scaling reduces towards $\gamma_{\text {eff }} \approx 0.42$. Figure $4(b)$ shows the compensated form, $\mathrm{Nu} / \mathrm{Ra}^{1 / 2}$, demonstrating that while the transitionally rough regime has a scaling near $\gamma_{\mathrm{eff}} \approx$ 0.55 , the fully rough regime clearly has a reduced scaling exponent.

Figure $4(\mathrm{c})$ shows the effective scaling exponent, $d \log _{10}(\mathrm{Nu}) / d \log _{10}(\mathrm{Ra})$, computed using the log-law formulas for smooth-wall and fully rough flows [Eqs. (3)-(6)]. This is also done using the curve fits of $\Delta U_{\text {fit }}^{+}$and $\Delta \Theta_{\text {fit }}^{+}$[Fig. 2(b)] in Eqs. (3) and (4), shown by the dotted lines. This figure is similar to Fig. 2(a) of Ref. [9], where for fully turbulent smooth-wall convection [solid line, Fig. 4(c)] we see the scaling exponent is in the range $0.35 \lesssim \gamma_{\text {eff }} \lesssim 0.42$. The transitionally rough regime has a much larger exponent, with $0.45 \lesssim \gamma_{\text {eff }} \lesssim 0.58$ for varying $k_{s} / L$ ratios and Ra. While the behavior of this exponent with Rayleigh number is dependent on the functional forms of $\Delta U_{\text {fit }}^{+}$ and $\Delta \Theta_{f i t}^{+}$, the important aspect is that the use of these fits shows an exponent close to 0.55 . As the flow enters the fully rough regime and $\Delta \Theta^{+}$becomes constant $\left(\mathrm{Ra} \approx 10^{9}\right.$ for $k_{s} / L=1.14 \times 10^{-1}$, black dashed line), the scaling exponent reduces to approximately $0.42 \lesssim \gamma_{\text {eff }} \lesssim 0.45$, consistent with Fig. 4(a), although still larger than that of the smooth-wall flow. Crucially, from Eqs. (5) and (6) we see that the rough-wall exponent must depend on the equivalent sand-grain roughness, $k_{S} / L$, as well as the temperature difference, $\Delta \Theta_{F R}^{+}$, making it distinct from the smooth-wall scaling exponent.

Discussion and conclusions. While we have used simple curve fits to obtain $\gamma_{\text {eff }} \approx 0.55$ in the transitionally rough regime, they show how large scaling exponents in the transitionally rough regime can be obtained [34,35,39]. As in Ref. [39], these large exponents do not indicate that the asymptotic ultimate regime has been obtained, as both viscous and thermal diffusivity effects are still important in the transitionally rough regime (referred to as regime I in Ref. [39]). It is only once the flow enters the fully rough regime (regime II in Ref. [39]), when the skin-friction coefficient is constant, that viscous actions become negligible and the scaling exponent reduces in value. Critically, however, thermal diffusivity effects will always remain important, as they do in the smooth wall. From Eq. (6), we see the fully rough centerline temperature and hence Nusselt number scales with the logarithm of Re, indicating that only for asymptotically large Ra does the heat transfer of rough-wall flows approach the asymptotic ultimate regime.

The origin for this major difference between momentum transfer and heat transfer in rough-wall shear flow lies in the pressure drag, which dominates the momentum transfer, but whose analog is absent for the heat transfer $[11,52,68]$. It is this absence which leads to an effective scaling $\mathrm{Nu} \sim$ $\mathrm{Ra}^{0.42}$ in the fully rough ultimate regime, rather than the upper bound exponent $1 / 2$. Our Nu versus $\mathrm{Ra}$ scaling prediction also seems to be consistent with recent rough-plate $\mathrm{RB}$ experiments in the Göttingen U-Boat facility, which in the ultimate regime yield an exponent of 0.43 for $\operatorname{Pr}=0.8$ and $\mathrm{Ra} \approx 10^{13}[69]$. 
We finally note that due to the limitations in fabrication every surface is rough to some degree $\left(k_{s} / L>0\right)$. For example, the Göttingen U-Boat system with $L=2.24 \mathrm{~m}$ uses either aluminum (HPCF-I) or copper (HPCF-II) top and bottom plates with average roughness heights of $R_{a} \approx$ $1.6 \mu \mathrm{m}$ and $0.2 \mu \mathrm{m}$, respectively [70]. At what Rayleigh number will this roughness become visible in the $\mathrm{Nu}(\mathrm{Ra})$ relation? Unfortunately, such an estimate is very difficult as it strongly depends on the prefactors and exact values of the scaling exponents. With the assumption of our above analysis, these surfaces could only be considered as fully smooth until $\mathrm{Ra} \approx 1 \times 10^{15}$ and $9 \times 10^{16}$ for the aluminum and copper plates, respectively, before becoming transitionally rough with an enhanced scaling exponent. These numbers should be taken with utmost care, because, as mentioned above, these estimates strongly depend on the coefficients in the Re-Ra relationship assumed previously, with the present coefficients $\left(\mathrm{Re}=0.7 \mathrm{Ra}^{0.5}\right)$ assuming fully turbulent boundary layers. If we use $\mathrm{Re}=0.346 \mathrm{Ra}^{0.443}$ from Grossmann-Lohse theory [9], then the corresponding critical Rayleigh numbers are $4 \times 10^{17}$ and $6 \times 10^{19}$, respectively. To obtain either set of values, the roughness is assumed to be hydrodynamically and thermodynamically smooth $\left(\Delta U^{+}=\Delta \Theta^{+}=0\right)$ until $k_{s}^{+}=4$, and that the plate surface equivalent sand-grain roughness is that of sinusoidal roughness, $k_{s} \approx 4.1 k$, where the sinusoidal semiamplitude is related to the mean roughness height by $k=2.46 R_{a}$ [53]. While there are uncertainties in the exact $\mathrm{Ra}$ values above, they provide some indication of the level of surface smoothness required in laboratory experiments to ensure the smooth-wall scaling exponent in the ultimate regime is observed. Regardless, as shown in Figs. 3 and 4, where the different line colors correspond to varying $k_{s} / L$, for sufficiently large Ra the surfaces will inevitably cease to be dynamically smooth.

Acknowledgments. The authors would like to gratefully acknowledge the financial support of the Australian Research Council through a Discovery Project (No. DP170102595).

[1] G. Ahlers, S. Grossmann, and D. Lohse, Heat transfer and large scale dynamics in turbulent RayleighBénard convection, Rev. Mod. Phys. 81, 503 (2009).

[2] D. Lohse and K.-Q. Xia, Small-scale properties of turbulent Rayleigh-Bénard convection, Annu. Rev. Fluid Mech. 42, 335 (2010).

[3] F. Chilla and J. Schumacher, New perspectives in turbulent Rayleigh-Bénard convection, Eur. Phys. J. E 35, 58 (2012).

[4] S. Grossmann and D. Lohse, Scaling in thermal convection: A unifying theory, J. Fluid. Mech. 407, 27 (2000).

[5] S. Grossmann and D. Lohse, Thermal Convection for Large Prandtl Number, Phys. Rev. Lett. 86, 3316 (2001).

[6] R. J. A. M. Stevens, E. P. van der Poel, S. Grossmann, and D. Lohse, The unifying theory of scaling in thermal convection: The updated prefactors, J. Fluid Mech. 730, 295 (2013).

[7] R. H. Kraichnan, Turbulent thermal convection at arbitrary Prandtl number, Phys. Fluids 5, 1374 (1962).

[8] E. A. Spiegel, Convection in stars, Annu. Rev. Astron. Astrophys. 9, 323 (1971).

[9] S. Grossmann and D. Lohse, Multiple scaling in the ultimate regime of thermal convection, Phys. Fluids 23, 045108 (2011).

[10] S. Grossmann and D. Lohse, Logarithmic temperature profiles in the ultimate regime of thermal convection, Phys. Fluids 24, 125103 (2012).

[11] P. R. Owen and W. R. Thomson, Heat transfer across rough surfaces, J. Fluid Mech. 15, 321 (1963).

[12] L. N. Howard, Heat transport by turbulent convection, J. Fluid. Mech. 17, 405 (1963).

[13] F. H. Busse, On Howard's upper bound for heat transport in turbulent convection, J. Fluid Mech. 37, 457 (1969).

[14] C. R. Doering and P. Constantin, Variational bounds on energy dissipation in incompressible flows: III. Convection, Phys. Rev. E 53, 5957 (1996). 
[15] X. He, D. Funfschilling, H. Nobach, E. Bodenschatz, and G. Ahlers, Transition to the Ultimate State of Turbulent Rayleigh-Bénard Convection, Phys. Rev. Lett. 108, 024502 (2012).

[16] X. He, D. Funfschilling, E. Bodenschatz, and G. Ahlers, Heat transport by turbulent Rayleigh-Bénard convection for $\operatorname{Pr}=0.8$ and $4 \times 10^{11}<\mathrm{Ra}<2 \times 10^{14}$ : Ultimate-state transition for aspect ratio $\Gamma=$ 1.00, New J. Phys. 14, 063030 (2012).

[17] G. Ahlers, E. Bodenschatz, and X. He, Logarithmic temperature profiles of turbulent Rayleigh-Bénard convection in the classical and ultimate state for a Prandtl number of 0.8, J. Fluid Mech. 758, 436 (2014).

[18] X. Chavanne, F. Chilla, B. Castaing, B. Hebral, B. Chabaud, and J. Chaussy, Observation of the Ultimate Regime in Rayleigh-Bénard Convection, Phys. Rev. Lett. 79, 3648 (1997).

[19] X. Chavanne, F. Chilla, B. Chabaud, B. Castaing, and B. Hebral, Turbulent Rayleigh-Bénard convection in gaseous and liquid He, Phys. Fluids 13, 1300 (2001).

[20] P. E. Roche, G. Gauthier, R. Kaiser, and J. Salort, On the triggering of the ultimate regime of convection, New J. Phys. 12, 085014 (2010).

[21] J. J. Niemela, L. Skrbek, K. R. Sreenivasan, and R. Donnelly, Turbulent convection at very high Rayleigh numbers, Nature (London) 404, 837 (2000).

[22] J. J. Niemela and K. R. Sreenivasan, Does confined turbulent convection ever attain the "asymptotic scaling" with 1/2 power? New J. Phys. 12, 115002 (2010).

[23] P. Urban, P. Hanzelka, V. Musilová, T. Králík, M. La Mantia, A. Srnka, and L. Skrbek, Heat transfer in cryogenic helium gas by turbulent Rayleigh-Bénard convection in a cylindrical cell of aspect ratio 1 , New J. Phys. 16, 053042 (2014).

[24] X. Zhu, V. Mathai, R. J. A. M. Stevens, R. Verzicco, and D. Lohse, Transition to the Ultimate Regime in Two-Dimensional Rayleigh-Bénard Convection, Phys. Rev. Lett. 120, 144502 (2018).

[25] D. Krug, X. Zhu, D. Chung, I. Marusic, R. Verzicco, and D. Lohse, Transition to ultimate RayleighBénard turbulence revealed through extended self-similarity scaling analysis of the temperature structure functions, J. Fluid Mech. 851, R3 (2018).

[26] Y. Shen, P. Tong, and K.-Q. Xia, Turbulent Convection Over Rough Surfaces, Phys. Rev. Lett. 76, 908 (1996).

[27] S. Ciliberto and C. Laroche, Random Roughness of Boundary Increases the Turbulent Convection Scaling Exponent, Phys. Rev. Lett. 82, 3998 (1999).

[28] Y. B. Du and P. Tong, Turbulent thermal convection in a cell with ordered rough boundaries, J. Fluid Mech. 407, 57 (2000).

[29] P. E. Roche, B. Castaing, B. Chabaud, and B. Hebral, Observation of the $1 / 2$ power law in RayleighBénard convection, Phys. Rev. E 63, 045303(R) (2001).

[30] X. L. Qiu, K.-Q. Xia, and P. Tong, Experimental study of velocity boundary layer near a rough conducting surface in turbulent natural convection, J. Turb. 6, N30 (2005).

[31] J. C. Tisserand, M. Creyssels, Y. Gasteuil, H. Pabiou, M. Gibert, B. Castaing, and F. Chilla, Comparison between rough and smooth plates within the same Rayleigh-Bénard cell, Phys. Fluids 23, 015105 (2011).

[32] J. Salort, O. Liot, E. Rusaouen, F. Seychelles, J.-C. Tisserand, M. Creyssels, B. Castaing, and F. Chilla, Thermal boundary layer near roughnesses in turbulent Rayleigh-Bénard convection: Flow structure and multistability, Phys. Fluids 26, 015112 (2014).

[33] P. Wei, T.-S. Chan, R. Ni, X.-Z. Zhao, and K.-Q. Xia, Heat transport properties of plates with smooth and rough surfaces in turbulent thermal convection, J. Fluid Mech. 740, 28 (2014).

[34] Y.-C. Xie and K.-Q. Xia, Turbulent thermal convection over rough plates with varying roughness geometries, J. Fluid Mech. 825, 573 (2017).

[35] M. J. Tummers and M. Steunebrink, Effect of surface roughness on heat transfer in Rayleigh-Bénard convection, Intl. J. Heat Mass Transfer 139, 1056 (2019).

[36] G. Stringano and R. Verzicco, Mean flow structure in thermal convection in a cylindrical cell of aspectratio one half, J. Fluid Mech. 548, 1 (2006).

[37] O. Shishkina and C. Wagner, Modelling the influence of wall roughness on heat transfer in thermal convection, J. Fluid Mech. 686, 568 (2011).

[38] S. Wagner and O. Shishkina, Heat flux enhancement by regular surface roughness in turbulent thermal convection, J. Fluid Mech. 763, 109 (2015). 
[39] X. Zhu, R. J. A. M. Stevens, R. Verzicco, and D. Lohse, Roughness-Facilitated Local 1/2 Scaling Does not Imply the Onset of the Ultimate Regime of Thermal Convection, Phys. Rev. Lett. 119, 154501 (2017).

[40] A. J. Smits, B. J. McKeon, and I. Marusic, High-Reynolds-number wall turbulence, Annu. Rev. Fluid Mech. 43, 353 (2011).

[41] A. J. Smits and I. Marusic, Wall-bounded turbulence, Phys. Today 66(9), 25 (2013).

[42] S. Grossmann, D. Lohse, and C. Sun, High Reynolds number Taylor-Couette turbulence, Annu. Rev. Fluid Mech 48, 53 (2016).

[43] F. H. Busse, Viewpoint: The twins of turbulence research, Physics 5, 4 (2012).

[44] B. Eckhardt, S. Grossmann, and D. Lohse, Torque scaling in turbulent Taylor-Couette flow between independently rotating cylinders, J. Fluid Mech. 581, 221 (2007).

[45] O. Cadot, Y. Couder, A. Daerr, S. Douady, and A. Tsinober, Energy injection in closed turbulent flows: Stirring through boundary layers versus inertial stirring, Phys. Rev. E 56, 427 (1997).

[46] T. H. van den Berg, C. R. Doering, D. Lohse, and D. P. Lathrop, Smooth and rough boundaries in turbulent Taylor-Couette flow, Phys. Rev. E 68, 036307 (2003).

[47] X. Zhu, R. A. Verschoof, D. Bakhuis, S. G. Huisman, R. Verzicco, C. Sun, and D. Lohse, Wall-roughness induces asymptotic ultimate turbulence, Nat. Phys. 14, 417 (2018).

[48] J. Nikuradse, Strömungsgesetze in rauhen rohren, Forsch. Arb. Ing.-Wes., 361 (1933); Laws of flow in rough pipes [English translation], NACA Tech. Memo. 1292 (1950).

[49] F. R. Hama, Boundary-layer characteristics for smooth and rough surfaces, Trans. Soc. Naval Arch. Mar. Engr. 62, 333 (1954).

[50] J. Jiménez, Turbulent flows over rough walls, Annu. Rev. Fluid Mech. 36, 173 (2004).

[51] B. Eckhardt, S. Grossmann, and D. Lohse, Fluxes and energy dissipation in thermal convection and shear flows, Europhys. Lett. 78, 24001 (2007).

[52] M. MacDonald, N. Hutchins, and D. Chung, Roughness effects in turbulent forced convection, J. Fluid Mech. 861, 138 (2019).

[53] L. Chan, M. MacDonald, D. Chung, N. Hutchins, and A. Ooi, A systematic investigation of roughness height and wavelength in turbulent pipe flow in the transitionally rough regime, J. Fluid Mech. 771, 743 (2015).

[54] D. Chung, L. Chan, M. MacDonald, N. Hutchins, and A. Ooi, A fast direct numerical simulation method for characterising hydraulic roughness, J. Fluid Mech. 773, 418 (2015).

[55] M. MacDonald, D. Chung, N. Hutchins, L. Chan, A. Ooi, and R. García-Mayoral, The minimal-span channel for rough-wall turbulent flows, J. Fluid Mech. 816, 5 (2017).

[56] B. A. Kader, Temperature and concentration profiles in fully turbulent boundary layers, Intl. J. Heat Mass Transfer 24, 1541 (1981).

[57] H. Kawamura, H. Abe, and Y. Matsuo, DNS of turbulent heat transfer in channel flow with respect to Reynolds and Prandtl number effects, Intl. J. Heat Fluid Fl. 20, 196 (1999).

[58] S. Pirozzoli, M. Bernardini, and P. Orlandi, Passive scalars in turbulent channel flow at high Reynolds number, J. Fluid Mech. 788, 614 (2016).

[59] A. M. Yaglom, Similarity laws for constant-pressure and pressure-gradient turbulent wall flows, Annu. Rev. Fluid Mech. 11, 505 (1979).

[60] Y. Miyake, K. Tsujimoto, and M. Nakaji, Direct numerical simulation of rough-wall heat transfer in a turbulent channel flow, Intl. J. Heat Fluid Flow 22, 237 (2001).

[61] S. Leonardi, P. Orlandi, and R. A. Antonia, Heat transfer in a turbulent channel flow with roughness, in Proceedings of the Fifth International Symposium on Turbulence and Shear Flow Phenomena (Munich, Germany, 2007), pp. 785-790.

[62] H. Schlichting, Experimentelle Untersuchungen zum Rauhigkeitsproblem, Ing. Arch. 7, 1 (1936); Experimental investigation of the problem of surface roughness [English translation], NACA Tech. Memo. 823 (1937).

[63] M. P. Schultz and K. A. Flack, Turbulent boundary layers on a systematically varied rough wall, Phys. Fluids 21, 015104 (2009).

[64] A. Busse, M. Thakkar, and N. D. Sandham, Reynolds-number dependence of the near-wall flow over irregular rough surfaces, J. Fluid Mech. 810, 196 (2017). 
[65] J. P. Monty, E. Dogan, R. Hanson, A. J. Scardino, B. Ganapathisubramani, and N. Hutchins, An assessment of the ship drag penalty arising from light calcareous tubeworm fouling, Biofouling 32, 451 (2016).

[66] W. M. Kays, M. E. Crawford, and B. Weigand, Convective Heat and Mass Transfer, 4th ed. (McGraw-Hill, New York, 2005).

[67] O. Shishkina, M. S. Emran, S. Grossmann, and D. Lohse, Scaling relations in large-Prandtl-number natural thermal convection, Phys. Rev. Fluids 2, 103502 (2017).

[68] T. Cebeci and P. Bradshaw, Physical and Computational Aspects of Convective Heat Transfer (SpringerVerlag, Berlin, 1984).

[69] E. Bodenschatz (private communication).

[70] G. Ahlers, D. Funfschilling, and E. Bodenschatz, Transitions in heat transport by turbulent convection at Rayleigh numbers up to $10^{15}$, New J. Phys. 11, 123001 (2009). 